\title{
SYNTHESIS AND CHARACTERIZATION OF PVA/AGNW COMPOSITES
}

\author{
Ramya. $\mathbf{P}^{1}$, Priya $\mathbf{L}^{2}$ \\ ${ }^{1}$ Department of Physics, CPGS, Jain University,Jayanagar 3rd Block,Bangalore-560011 \\ ramya.perecharal@gmail.com \\ ${ }^{2}$ Department of Physics, LRG Government College for women,Tirupur- 641604 \\ svl.priya1@gmail.com
}

\begin{abstract}
The influence of silver nanowires and (AgNWs) on the structure, morphology and electrical properties of Polyvinyl Alcohol (PVA) has been studied in the present work. PVA/AgNW samples have been prepared by dissolving PVA in AgNW solution and the thin films has been prepared by solution casting method. Structural and morphological properties have been studied from XRD and FESEM. AgNWs affected the signature peak of PVA at 20o by breaking the hydrogen bonding and OH groups. All the fcc peaks of $\mathrm{Ag}$ are retained at same positions in all the composites. FESEM of the composites shows that AgNWs are well embedded in the PVA matrix. UV-Vis spectroscopy shows that the optical band gap of the composites is less than that of the PVA. $D C$ conductivity results show that the conductivity of the composites has increased by nearly four orders than pure PVA. Best are obtained only till 15\% of AgNW doping concentration. After 15\% AgNWs got aggregated in the matrix and conductivity decreased in the composite containing $20 \%$ AgNWs.
\end{abstract}

\section{INTRODUCTION:}

Polymer based metal nanocomposites are attracting a considerable interest in the recent times as they blend the mechanical properties, processability, durability of polymers and the optical, thermal and electrical properties of metals[1-4]. It is a well known fact that the properties of nanostructures strongly depends on their size and shape and the polymers acts as best hosts for metal nanostructures. Opto-electrical properties of silver nanowires (AgNWs) are attracting the attention of the researchers towards the applications like liquid crystal displays, OLEDs, solar cells [5]. PVA is water soluble extensively investigated transparent polymer for nanofillers and behaves in different ways with different fillers. PVA is a material where its physicochemical properties are dependent on the degree of polymerization, hydrolysis, and distribution of its hydroxyl groups $(\mathrm{OH})[6]$.

In the present work PVA/ AgNW (PVAW) composites has been prepared by adding AgNWs in to water solution containing PVA. Homogeneous film were then obtained by solution casting technique after evaporation of water. The samples were characterized by XRD, FESEM, UV- VIS spectroscopy for their Structure and morphology. DC conductivity of the samples has been done by two probe technique in the temperature range of $298 \mathrm{~K}$ to $398 \mathrm{~K}$.

\section{EXPERIMENTAL DETAILS:}

Materials Used: Silver nitrate $\left(\mathrm{AgNO}_{3}\right)$, trisodium citrate, Polyvinyl alcohol (PVA) were obtained from SD Fine chem. Limited. Aniline, Sodium hydroxide $(\mathrm{NaOH})$, Hydrochloric acid were purchased from Merc Specialities private limited and Ammonium per sulphate from Fisher Scientific. All the chemicals were used as received.

Preparation of AgNWs: AgNWs were synthesized by Seedless, Surfactant less wet chemical method[7]. The synthesis involves two silver solutions A and B. Beaker A consisted of $100 \mathrm{ml}$ of deionised water, $2 \mu \mathrm{l}$ of $1 \mathrm{M} \mathrm{NaOH}$ and $40 \mu \mathrm{l} 0.1 \mathrm{M} \mathrm{AgNO}_{3}$. This solution was brought to boil with rapid stirring and in this $5 \mathrm{ml}$ of $0.01 \mathrm{M}$ trisodium citrate was quickly added. Then the solution was allowed to boil for another 10min. Concomitantly, Solution B was prepared by adding $150 \mathrm{ml}$ deionised water, $2 \mu \mathrm{l} 1 \mathrm{M} \mathrm{NaOH}$ and $20 \mu \mathrm{l}$ $0.1 \mathrm{M} \mathrm{AgNO}_{3}$ and bringing this to boil . Solution B was added to solution $\mathrm{A}$ and the mixture was allowed to evaporate till the solution becomes $\sim 75$ to $100 \mathrm{ml}$.

Preparation of PVA/ AgNW (PVAW) composites: Appropriate amount of PVA has been dissolved in AgNW solution in deionised water. This mixture has been stirred vigorously for about 2 hours for homogenous distribution of AgNWs into the matrix. Then uniform films of the composites have been formed by solution casting technique by evaporation of the solvent.

\section{CHARACTERIZATION:}

X-Ray diffraction (XRD) of the composites was done to know their crystal structure through Rigaku Smart lab X-ray diffractometer equipped with a $\mathrm{Cu}-\mathrm{K} \alpha$ source. Morphologies of samples were determined with Carl Zeiss Ultra 55 Field Emission Scanning Electron Microscope. UV-Vis spectra of the aqueous dispersions were obtained from 1800 Shimadzu $\mathrm{UV}-\mathrm{Vis}$ spectrophotometer. Electrical properties of PVA 
and composites were investigated from lab set up two probe method.

Table: 1 Sample codes and optical band gaps of PVA and the composites.

\begin{tabular}{|l|l|l|}
\hline Sample Name & Sample code & $\begin{array}{l}\text { Optical band } \\
\text { gap (ev) Eg }\end{array}$ \\
\hline $\begin{array}{l}\text { Polyvinyl } \\
\text { Alcohol }\end{array}$ & PVA & 3.5 \\
\hline $\begin{array}{l}\text { PVA/AgNW } \\
5 \%\end{array}$ & PVAW 5 & 2.1 \\
\hline $\begin{array}{l}\text { PVA/AgNW } \\
10 \%\end{array}$ & PVAW10 & 2 \\
\hline $\begin{array}{l}\text { PVA/AgNW } \\
12 \%\end{array}$ & PVAW12 & 3.25 \\
\hline $\begin{array}{l}\text { PVA/AgNW } \\
15 \%\end{array}$ & PVAW15 & 2.1 \\
\hline $\begin{array}{l}\text { PVA/AgNW } \\
20 \%\end{array}$ & PVAW20 & 3.5 \\
\hline
\end{tabular}

\section{RESULTS AND DISCUSSION:}

XRD: From Figure 1 XRD of PVA shows a sharp peak at $2 \theta=20^{\circ}$ indicating that most of PVA molecules are arranged in (101) plane.

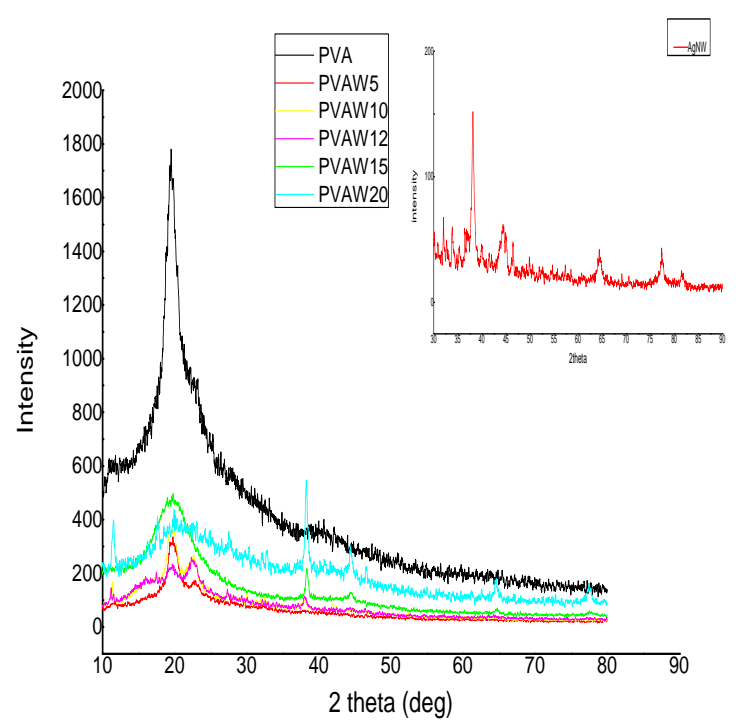

Figure: 1 XRD of PVA and PVAW samples with XRD of $\mathrm{AgNW}$ in the inset.

This peak shows the semi crystalline nature of PVA due to presence of strong inter-molecular and intra-molecular hydrogen bonding between the PVA chains [8]. Other minor peak at $23^{\circ}$ might be due to minor crystallites or due to impurities. XRD of AgNW shows peaks at $2 \theta=38^{\circ}, 45^{\circ}, 65^{\circ}$ and $78^{\circ}$ corresponding to (111), (200), (220) and (311) planes respectively corresponding to fcc crystal structure (JCPDS, File No. 04-0783).

In the composites the intensity of characteristic peak of PVA at $2 \theta=20^{\circ}$ has been reduced by increasing the concentration of AgNWs in to PVA matrix and the intensity of peak at
$2 \theta=23^{\circ}$ has increased gradually. In PVAW20 both the peaks at $2 \theta=20^{\circ}$ and $23^{\circ}$ combined to a broad peak. The incorporation of AgNWs into the PVA matrix would have disturbed the hydrogen bonding and $\mathrm{OH}$ group of PVA, which resulted in the breakage of polymer chains. This might be the reason for the decrease in intensity of peak at $20^{\circ}$ [9]. The breakage in the molecular chains lead the molecules free, so that they might rotate in the direction (200) because of which the intensity at $23^{\circ}$ would have increased.

All the composites shows the peaks corresponding to the fcc crystal structure of $\mathrm{Ag}$ and the peak intensities increased with the increase in Ag percentage in the composites. As there is no chemical reaction between PVA and AgNWs, the peaks of cubic structure of $\mathrm{Ag}$ are at the same positions. This can be observed from the FESEM images.

FESEM: Figure 2b) shows the FESEM image of PVAW10 in which a well embedded AgNW network is seen clearly. The nanowires are well dispersed in the matrix of PVA. Some wires even deeply immersed in the matrix with a small portion of the wire protruded out, which shows the random nature of dispersion.

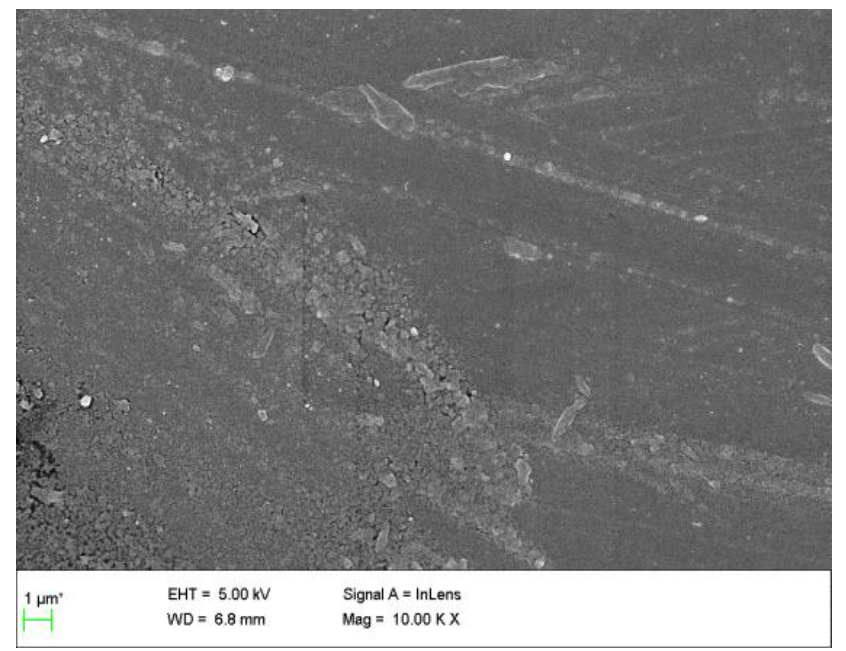

(a) FESEM of PVA

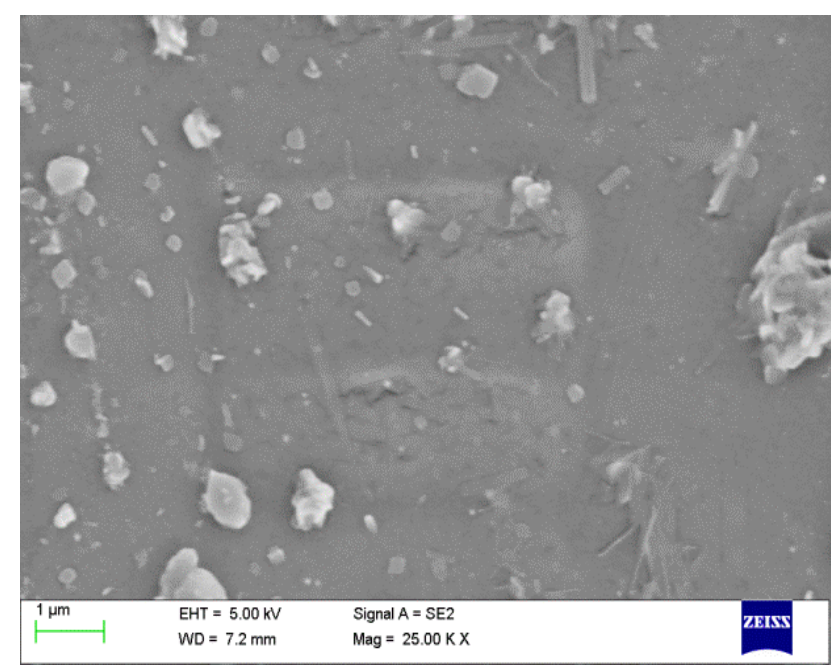

(b) FESEM of PVAW 10 


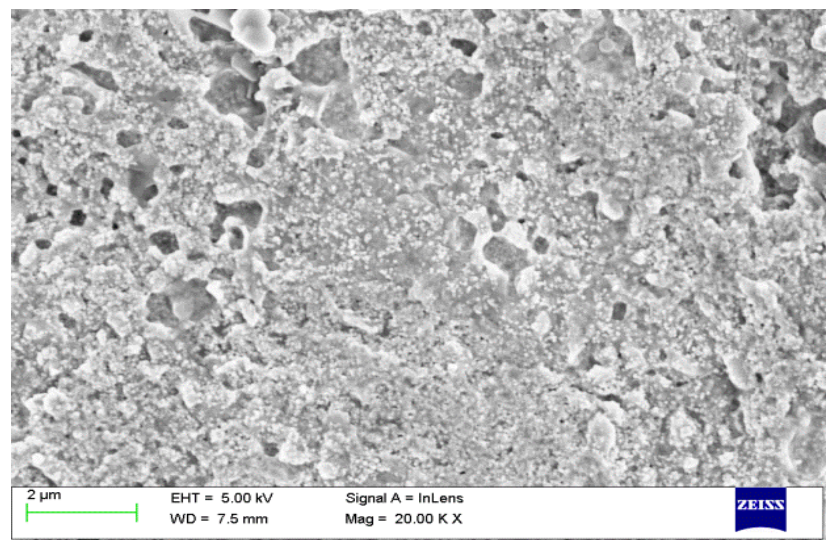

(c) FESEM of PVAW 20

Figure: 2 FESEM images of PVA and the composites

In the image of PVAW 20 there is a lot of $\mathrm{Ag}$ cluster formation because of which the wires are not seen. The dispersion of nanowires is also not seen.

\section{UV-Vis Spectroscopy:}

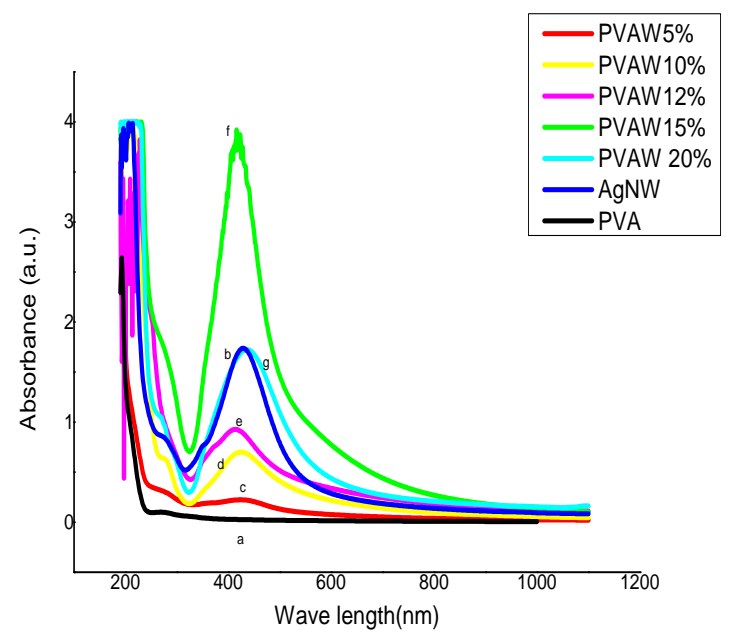

Figure: 2 UV-Vis Spectroscopy of PVA, AgNW and the composites

UV-Visible spectroscopy of PVA, AgNW and the composites is given in the figure: 2. PVA (curve 'a') has a peak at $273 \mathrm{~nm}$ which may be due to the $\mathrm{n} \rightarrow \pi^{*}$ transitions of the $\mathrm{C}=\mathrm{O}$ group of PVA [9]. There is no other peak in the entire visible region. AgNWs (' $b$ ' curve) in this figure shows an absorption band at $427 \mathrm{~nm}$ which is due to surface plasmon resonance of silver ions that collectively oscillates due to resonance with the incident electromagnetic radiation (visible light). There is a shoulder peak at $352 \mathrm{~nm}$ in the same curves of $\mathrm{AgNW}$, which is due to the transverse plasmon resonance of nanowires of $\mathrm{Ag}[10]$.

In the composites, absorption peak at $273 \mathrm{~nm}$ is due to PVA matrix and the shoulder peak at $352 \mathrm{~nm}$ is sustained in all the composites. The SPR peak due to Ag ions has been shifted to lower wavelength from $427 \mathrm{~nm}$ in PVAW5 to $412 \mathrm{~nm}$ in PVAW15 again shifted to the same position $427 \mathrm{~nm}$ in PVAW20. Shifting of the peak to lower wavelength may be due to decrease in the diameter of $\mathrm{AgNW}$ after doping into the matrix [11]. Good dispersion of wires has observed in the composites till PVAW15. This can be confirmed with the increase in intensity of absorption peak up to PVAW15. However it is well known fact that the absorption of nanostructures depends mainly on their size. As the diameter of the wires is reduced, the intensity of the absorption peak increased and a corresponding shift towards the lower wavelengths has been observed.

In PVAW20, because of increased Ag concentration the wire structure has been damaged after doping into the matrix and the nanostructures not dispersed well in the matrix which is also confirmed in FESEM of PVAW20. The absorption peak may have shifted again to the higher wavelength side with a less intensified absorption due to this reason.

Optical energy band gap of Pure PVA and the composites has been obtained using Tauc relation. The values of optical band gaps has determined by extrapolating the lines fitted in the plots of hv versus $(\alpha h v)^{2}$ where $\alpha$ is the absorption coefficient and hv is the energy of incident photon[12]. The values are tabulated in the Table no. 1

Optical band gap has decreased from 3.5ev in PVA to 2.1ev in the composites. This reduction in value of optical band gap may be due to the formation of chemical bonds between PVA chains and AgNWs responsible for generation of localized states between HOMO and LUMO energy bands in PVA. The AgNWs has formed connecting networks in the PVA matrix which is evident from the FESEM of PVAW10. Embedding of AgNWs in the PVA matrix would have generated new energy levels between the HOMO and LUMO which made the energy transitions possible at the lower energies. In PVAW20 such networks are absent and the AgNWs has agglomerated in the matrix. This might be the reason for the increment of $\mathrm{E}_{\mathrm{g}}$ to $3.5 \mathrm{ev}$ in PVAW20.

\section{DC conductivity:}

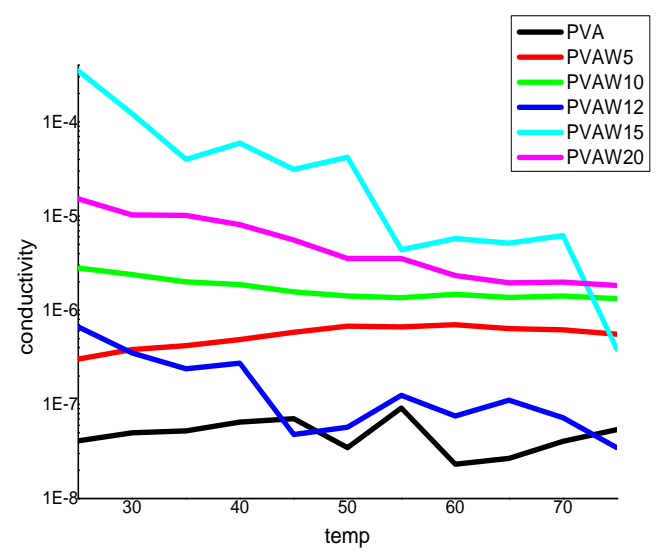

Figure: 4 DC conductivity of PVA and the composites

Figure: 4 shows the variation of conductivity of the samples with respect to temperature. Pure PVA has conductivity of 4.10549E-8 S/cm at $298 \mathrm{~K}$. By introducing AgNWs in to PVA matrix conductivity has increased by two orders 
minimum in PVAW5. With the increase in AgNW concentration in the matrix the conductivity has improved and PVAW15 shows maximum conductance of approximately $3.5 \mathrm{E}-4 \mathrm{~S} / \mathrm{cm}$. That is conductivity has improved by 4 orders magnitude when compared to pure PVA.

Increase in conductivity in the composites may be due to the formation of charge transfer complexes in the polymer networks with the introduction of AgNWs in the matrix. With the formation of charge transfer complexes in semi crystalline polymers like PVA, the barrier height between the trapping sites reduces which in turn provides a connecting path in the amorphous regions of PVA and hence the conductivity enhances [13]. The Ag ions fill the free volume holes and occupy free interstitial places between the polymer chains in the amorphous phase and link these chains by charge exchange process between the nanowires and the PVA chains. Further this complex formation increases with the doping concentrations of AgNWs. Because of this reason conductivity has increased with the increase in AgNWs percentage in PVA

Conductivity of PVAW 20 is less compared to PVAW 15, which might be due to aggregation of $\mathrm{Ag}$ in the matrix. Because of cluster formation AgNWs could not provide the connecting path in the amorphous PVA matrix which resulted in low conductivity. Aggregation of AgNWs in PVAW20 is evident from FESEM images. Also, the optical band gap of PVAW 20 supports these results.

\section{CONCLUSION}

PVA/AgNW composites have been synthesized and characterized for their structural, morphological and electrical properties. Addition of AgNWs into PVA has affected its structure which is evident from reduction of intensity of signature peak of PVA at $20^{\circ}$. FESEM image of PVAW 10 shows the well dispersion of AgNWs in the polymer matrix. UV-Vis spectroscopy of the samples shows a blue shift in the SPR absorption peak of Ag ions, which may be due to reduction of diameter of AgNWs. There is a reduction in optical band gap till PVAW15 which also supports the conductivity of the samples. Conductivity in the composites has improved nearly by 4 orders magnitude by the introduction of $15 \% \mathrm{AgNWs}$ in to PVA.

\section{REFERENCES:}

[1]. A. Nimrodh Ananth and S. Umapathy, "On the optical and thermal properties of in situ/ex situ reduced AgNP's / PVA composites and its role as a simple SPR-based protein sensor," Applied Nanoscience, vol. 1, no. 2, pp. 87-96, 2011.

[2]. G. Nesher, G. Marom, and D. Avnir, "Metal-polymer composites: synthesis and characterization of polyaniline and other polymer at Silver compositions," Chemistry ofMaterials, vol. 20, no. 13, pp. 4425-4432, 2008.

[3]. P.-H.Wang, Y.-Z.Wu, andQ.-R. Zhu, "Polymermetal composite particles: polymer core and metal shell,"
Journal of Materials Science Letters, vol. 21, no. 23, pp. 1825-1828, 2002.

[4]. S. Cl'emenson, P. Alcouffe, L. David, and E. Espuche, "Structure and morphology of membranes prepared from polyvinyl alcohol and silver nitrate: influence of the annealing treatment and of the film thickness," Desalination, vol. 200, no. 1-3, pp. 437-439, 2006.

[5]. Choong-Heui Chung,et.al., "Solution-Processed Flexible Transparent Conductors Composed of Silver Nanowire Networks Embedded in Indium Tin Oxide Nanoparticle Matrices" Nano Research 2012, Volume 5, Issue 11, pp 805-814

[6]. Asmalina Mohamed Saat and Mohd Rafie Johan "Effect of Phosphoric Acid Concentration on the Optical Properties of Partially Phosphorylated PVA Complexes" International Journal of Polymer Science, Volume 2014, Article ID 495875.

[7]. K. K. Caswell et al., "Seedless, Surfactantless Wet Chemical Synthesis of Silver Nanowires." Nano Lettes., Vol. 3, No. 5, 2003

[8]. H.N.Chandrakala et al.,'Optical properties and structural characteristics of zinc oxide, cerium oxide doped polyvinyl alcohol films", Journal of Alloys and Compounds 586, 2014, 333-342.

[9]. Rishi Pal Chahal et al., " $\gamma$-Irradiated PVA/Ag nanocomposite films: Materials for optical applications" Journal of Alloys and Compounds 538, 2012 212-219

[10].Marco Bernabò et al., "Polymer Nanocomposites Containing Anisotropic Metal Nanostructures as Internal Strain Indicators" Materials 2010, 3, 14611477

[11]. Shawkat Salameh Gasaymeh et al., Synthesis and Characterization of Silver/Polyvinilpirrolidone (Ag/PVP) Nanoparticles Using Gamma Irradiation Techniques" African Physical Review, 2010 4:0006 31-41

[12].E. A. Davis and N. F.Mott, "Conduction in noncrystalline systems Conductivity, optical absorption and photoconductivity in amorphous semiconductors," PhilosophicalMagazine, vol. 22, pp. 903-922, 1970.

[13].R F Bhajantri "Effect of barium chloride doping on PVA microstructure: positron annihilation study" 2007 Appl. Phys. A 87 797-805 